\title{
Fachinformationen der
}

\section{Blätter der Wohlfahrtspflege}

Blätter der Wohlfahrtspflege - Deutsche Zeitschrift für Soziale Arbeit - www.bdw.nomos.de

\section{Paritätischer sieht sozialen Zusammenhalt gefährdet}

Deutliche Defizite der Bundesregierung in der Sozialpolitik stellt der Paritätische Gesamtverband in seinem aktuellen Gutachten zur sozialen Lage in Deutschland fest. Die Analyse sozioökonomischer Indikatoren belege eine stetig tiefer werdende soziale Spaltung. Die im vergangenen Jahr umgesetzten Gesetzesvorhaben seien in der Gesamtheit nicht geeignet gewesen, dieser Entwicklung substantiell entgegenzuwirken, so das Ergebnis des Gutachtens. Durch Maßnahmen wie die umstrittene Rentenreform sei die Spaltung sogar noch verschärft worden. Der Verband fordert durchgreifende sozialpolitische Reformen insbesondere zur Bekämpfung von Armut und Langzeitarbeitslosigkeit. Zur Finanzierung sei eine stärkere Besteuerung sehr großer Einkommen und Vermögen erforderlich. Kern des Gutachtens ist eine Analyse und Bewertung der im Berichtsjahr 2014 umgesetzten sozialpolitischen Gesetzesvorhaben - von der Pflegereform über das Rentenpaket bis hin zu Veränderungen im Staatsangehörigkeitsrecht. Der Bericht steht auf der Webseite der Organisation kostenlos zur Verfügung. www.der-paritaetische.de

\section{Zusammenarbeit mit Familien und Netzwerkpartnern in der Migrationsgesellschaft}

Im Projekt des Deutschen Roten Kreuzes »stark für Erfolg - Begleitung von Kind und Familie bei Bildungsübergängen" wurden von 2012 bis 2014 an bundesweit 30 DRK-Standorten Bildungspartnerschaften mit Familien aufgebaut (vgl. Blätter der Wohlfahrtspflege 3/2014 und 5/2014). Mit über 17.000 Teilnehmenden aus 33 Ländern und über 93.000 Beratungen zeigt es, wie Familien mit Zuwanderungsgeschichte erfolgreich erreicht und gleichberechtigt beteiligt werden können und wie Kinder und Jugendliche in ihrem Bildungsverlauf wirksame Unterstützung erfahren können. Anhand verschiedener Beispiele werden nun in einem Praxishandbuch erfolgversprechende Ansätze für die Zusammenarbeit mit Eltern und Netzwerkpartnern im interkulturellen Kontext vorgestellt. Die 128-seitige Veröffentlichung »Bildungsübergänge gemeinsam gestalten « kann kostenlos aus dem Internet heruntergeladen werden. http://drk-wohlfahrt.del veroeffentlichungen.html

\section{Der Allgemeine Soziale Dienst unter Druck}

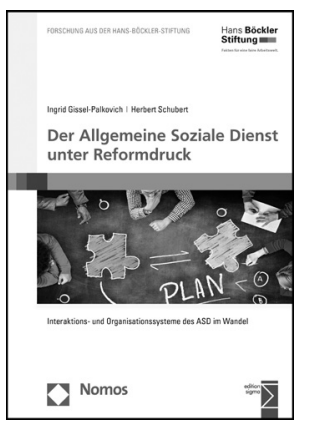

Seit Jahren steht der Allgemeine Soziale Dienst (ASD) als das Kernstück des kommunalen sozialen Dienstleistungsnetzes unter einem erheblichen Entwicklungsdruck: Er muss steigende Fallzahlen und erhöhte Fallkomplexität bewältigen, neue fachliche Trends und gesellschaftliche Bedarfe flexibel aufgreifen, dem Ruf nach ganzheitlichen, einheitlichen und effizienten Hilfsleistungen folgen und dabei Organisationsreformen nach Maßgabe eines modernen Verwaltungsverständnisses durchführen. Und das alles vor dem Hintergrund einer anhaltend schwierigen kommunalen Haushaltslage. Ein neues Buch stellt in kompakter Form die Frage ins Zentrum, wie diese Vielfalt von teilweise widerstreitenden Zielen bewältigt werden kann. Auf der Basis einer soliden theoretischen Fundierung und gestützt auf eine breite qualitative Empirie untersuchen die Autoren, welche Faktoren ausschlaggebend sind, um eine "gelingende ASD-Praxis" sicherzustellen. Dabei entsteht eine Typologie von Reformansätzen, anhand derer gangbare und erfolgversprechende Entwicklungswege deutlich werden. Neben der Perspektive der Akteure kommt auch jene der Adressaten zu Sprache, und Erfolge werden ebenso reflektiert wie Widersprüche und Hemmnisse. Gissel-Palkovich, Herbert Schubert: Der Allgemeine Soziale Dienst unter Reformdruck. Interaktions- und Organisationssysteme des ASD im Wandel. Nomos Verlagsgesellschaft, Baden-Baden 2015. 220 Seiten. 18,90 Euro. ISBN 978-3-8487-2063-7.

\section{Arbeitshilfe zur Gewaltprävention}

Der Paritätische Wohlfahrtsverband Hessen hat Hinweise und Musterformulare für Träger sozialer Einrichtungen zur Gewaltprävention vorgelegt. Kinder und Jugendliche sowie Menschen mit Pflegebedarf oder Behinderungen müssen in besonderem Maße vor jeglicher Form der Gewalt geschützt werden. Um diesem Schutzbedürfnis Rechnung zu tragen, sind in den vergangenen Jahren eine Reihe von neuen gesetzlichen Bestimmungen eingeführt worden. Die neue Arbeitshilfe richtet sich an Arbeitgeber in der Sozialen Arbeit und reicht von Musterformularen zur Beantragung von Führungszeugnissen über Verpflichtungserklärungen für Beschäftigte in der Kinder- und Jugendhilfe sowie in der Altenhilfe bis hin zu wichtigen Aspekten des Datenschutzes. Als barrierefreies PDF-Dokument steht die Arbeitshilfe zum kostenlosen Herunterladen bereit. www.paritaet-hessen.org

\section{Betriebliche Kinderbetreuung weiterhin sehr selten}

Betriebskindergärten sind nach wie vor eine Rarität. Nicht einmal ein Prozent aller außer Haus betreuten Kinder besuchen eine betriebliche Kita. Das ergibt eine Auswertung des Wirtschafts- und Sozialwissenschaftlichen Instituts (WSI) in der Hans-Böckler-Stiftung aufgrund einer Vollerhebung des Statistischen 
Bundesamts. Demnach beträgt der Anteil der betrieblich betreuten Kinder an allen in Tageseinrichtungen betreuten Kindern lediglich 0,9 Prozent. 2014 gab es laut Bundesamt in der Bundesrepublik insgesamt 53.415 Kindertagesstätten. Zum Vergleich: 2012 existierten in ganz Deutschland 586 und im vergangenen Jahr 668 »Tagesstätten für Kinder von Betriebsangehörigen " - bei insgesamt 3,6 Millionen Unternehmen.

http://boeckler.de

\section{Neue Dörfer braucht das Land}

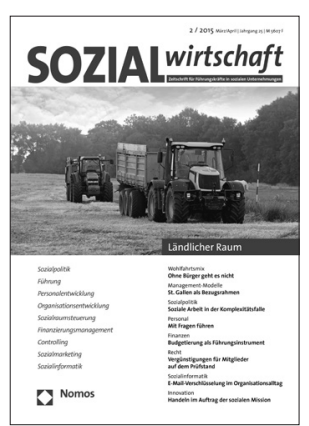

Die Zeitschrift SOZIALwirtschaft widmet sich in ihrer Ausgabe 2/2015 dem ländlichen Raum. Unter anderem geht es darum, wie die soziale und gesundheitliche Versorgung in der Fläche gewährleistet werden kann und welche Aufgaben sich dabei für Wohlfahrtsverbänden und Sozialunternehmen stellen. Fachlich interessante Konzepte wären beispielsweise Bauernhöfe, die ganz oder teilweise mit Außenarbeitsplätzen von Werkstätten für behinderte Menschen betrieben werden oder die Begleitung von Familien auf dem Lande, die pflegebedürftige oder psychisch kranke Menschen aufgenommen haben.

www.sozialwirtschaft.nomos.de

\section{Assistenten auf vier Beinen}

Blinden Menschen fällt es in unserer lauten und hektischen Gesellschaft nicht einfach sich zurechtzufinden. Es wurden schon zahlreiche Hilfsmittel entwickelt die das Leben eines sehbeeinträchtigten Menschen erleichtern sollen. Eine besondere Stellung dabei nimmt nach wie vor der Blindenführhund ein. Ein neues Buch stellt dazu die Ergebnisse einer Bachelorarbeit vor. Auf Grundlage der Fachliteratur und qualitativer Interviews und Feldbeobachtungen wird das Verhalten der Mitmenschen gegenüber einem blinden Menschen mit Blindenführhund

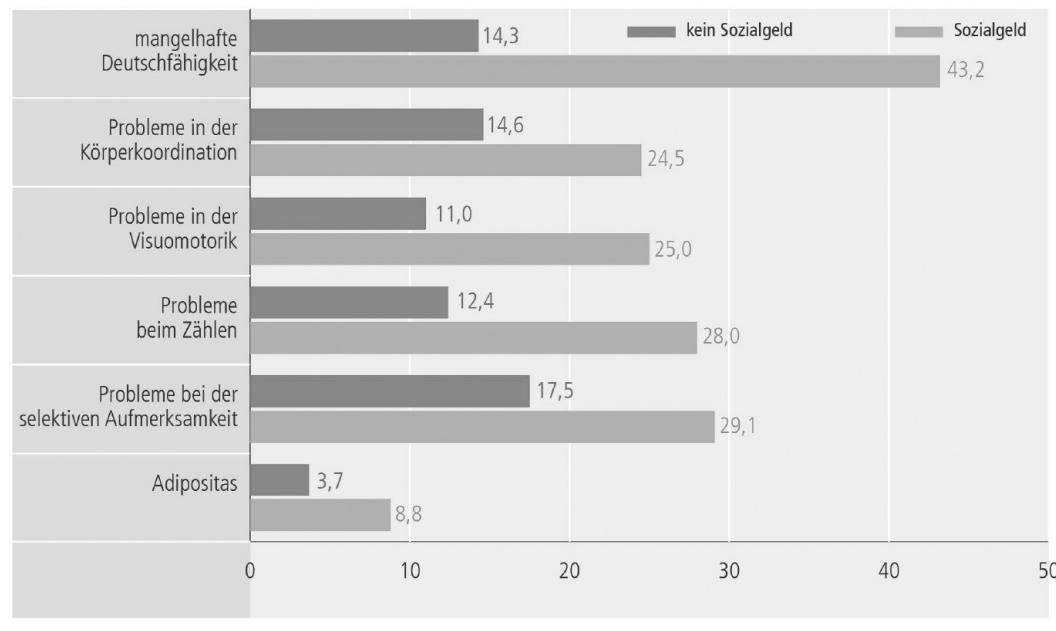

Quelle: Schuleingangsuntersuchung Mülheim an der Ruhr 2009/2010 bis 2012/2013, eigene Berechnung und Darstellung.

(c) Bertelsmann Stiftung und ZEFIR 2015, mit finanzieller Unterstützung des Landes NRW und des Europäischen Sozialfonds.

Kinderarmut ist kein Randphänomen, sondern betrifft jedes fünfte Kind unter drei Jahren, wie eine Studie der Bertelsmann Stiftung und der Ruhr-Universität Bochum für Nordrhein-Westfalen festgestellt hat. Für die Mehrheit der armen sechsjährigen Kinder ist der SGB II-Bezug auch ein Dauerzustand, der das Aufwachsen bestimmt. Diese Kinder sind in einem hohen Maße unterstützungsbedürftig, wenn sie nicht dauerhaft zurückgelassen werden sollen. Die mit Hilfe der Ergebnisse von Schuleingangsuntersuchungen und Daten aus der SGB II-Statistik ermittelten Fakten sind in einem 67-seitigen Werkstattbericht zusammengefasst, der kostenlos aus dem Internet heruntergeladen werden kann.

www.zefir.ruhr-uni-bochum.de

gezeigt. Zudem verweist die Autorin auf die Rolle der Sozialen Arbeit, die im Rahmen der Öffentlichkeits- und Aufklärungsarbeit, aber auch in Hinblick auf die Stadtentwicklung noch Handlungsbedarf hat.

Madleen Morina: Die Rolle von Assistenzhunden bei der Inklusion von Menschen mit Behinderung in die Gesellschaft. Der Alltag von blinden Menschen in der Metropolregion Nürnberg Untersuchung und Diskussion über den Einsatz von Blindenführhunden. Disserta Verlag, Hamburg 2014. 254 Seiten. 44,99 Euro. ISBN 978-3954258666.

\section{Rückgang beim Bundesfreiwilligendienst}

Noch nie waren die Chancen so schlecht, einen Platz im Bundesfreiwilligendienst zu erhalten. Obwohl es immer noch viele Bewerber gibt, ist die Anzahl der Dienstantritte um mehr als ein Viertel zurückgegangen. Seit der Einführung des Bundesfreiwilligendienstes 2011 haben sich fast 160.000 Männer und Frauen beteiligt und sich in einem sozialen Projekt engagiert.
Der Bundesfreiwilligendienst sollte eigentlich ein Freiwilligendienst für alle Altersgruppen sein; doch in der praktischen Umsetzung wird er laut Bundesamt für Familie und zivilgesellschaftliche Aufgaben immer mehr zu einem weiteren Jugendfreiwilligendienst. Die Bundesregierung stellt für den Bundesfreiwilligendienst im Haushalt jährlich 167 Millionen Euro bereit. Letztes Jahr reichte dieser Betrag jedoch nicht aus, so dass der Bundestag zusätzliche elf Millionen Euro freigegeben hat. Dieser Betrag wird dem Haushalt 2015 vermutlich nicht zur Verfügung gestellt, so dass mit einem weiteren Rückgang der Zahlen zu rechnen ist. www.bafza.de

\section{Anstellungsträger sollten ihre Attraktivität hervorheben}

Unverändert fußt die Entscheidung für einen sozialen Beruf auf dem Wunsch, Menschen zu helfen. Für die Wahl des konkreten Arbeitgebers sind jedoch auch Bezahlung und Rahmenbedingungen entscheidend. $\mathrm{Zu}$ diesem Ergebnis kommt eine Untersuchung der 
Hochschule für Technik, Wirtschaft und Kultur Leipzig im Auftrag des Paritätischen Wohlfahrtsverbandes Sachsen, für die Auszubildende und Studierende sozialer Berufe befragt wurden. Die Untersuchung formuliert anhand der Ergebnisse auch Handlungsempfehlungen. Neben abwechslungsreichen Aufgaben spielen die persönlichen Entwicklungsmöglichkeiten eine wesentliche Rolle. Arbeitgeber müssen die Motivation der Nachwuchsfachkräfte im beruflichen Alltag besser aufgreifen. Soziale Einrichtungen sind gefordert, die eigene Arbeitgeberattraktivität konkreter als bisher darzustellen und dafür Ressourcen einzuplanen. Die Untersuchung steht im Internet zur Verfügung.

www.parisax.de

\section{Stiftung Mitarbeit vergibt Starthilfeförderungen}

Die Stiftung Mitarbeit vergibt viermal im Jahr Starthilfezuschüsse an kleinere lokale Organisationen mit geringen eigenen finanziellen und personellen Ressourcen sowie an neue Initiativen und Gruppen, die in den Bereichen Soziales, Politik, (Erwachsenen-)Bildung, Kultur, Umweltschutz, Gesundheit und
Kommunales innovativ tätig sind. Die nächsten Termine für Bewerbungen in diesem Jahr sind der 24. August und der 2. November 2015. Die Starthilfeförderung richtet sich an Gruppen und Initiativen, denen sonst keine oder nur unzureichende Fördermöglichkeiten offen stehen. Ein Projekt kann in der Regel nur einmal mit einem Höchstbetrag von 500 Euro bedacht werden. Gefördert werden können Aktivitäten, die auf freiwilligem und ideellem Engagement beruhen und beispielsweise dazu beitragen, gesellschaftliche Konflikte auf demokratischem Wege zu lösen und persönliche Eigeninitiative und Handlungskompetenz zu stärken. Wesentlich ist, dass die förderfähigen Aktionen aufzeigen, wie Zusammenschlüsse von freiwillig engagierten Menschen das Leben in unserer Gesellschaft mitgestalten können.

www.mitarbeit.de

\section{Bundesregierung plant Leitfaden zur Inklusion}

Im Rahmen der UN-Behindertenrechtskonvention erstellt die Bundesregierung derzeit einen Leitfaden zur »konsequenten Einbeziehung der Belange behinderter Menschen in die Gesetzgebungs-, Verwaltungs- und sonstigen Vorhaben der Ministerien«. Das teilt sie in ihrer Antwort (18/4359) auf eine Kleine Anfrage der Bundestagsfraktion »Die Linke« mit. Zuständig für den Leitfaden ist das Bundesministerium Arbeit und Soziales, beteiligt werden die Anlaufstellen (»Focal Points«) der Bundesministerien zur Umsetzung der UN-Behindertenrechtskonvention und die Schwerbehindertenvertretungen der Bundesministerien. Künftig solle anhand dieses Leitfadens frühzeitig erkannt werden, welche Auswirkungen für Behinderte im Vergleich zu Menschen ohne Behinderung zu erwarten seien, schreibt die Bundesregierung. Zudem beinhalte der Leitfaden praktische Hilfen für die Beteiligung von Behindertenorganisationen. www.bundestag.de

\section{Kostenloses Praxis-Handbuch zur Mitarbeiterbindung}

Nur Arbeitgeber, die ihren Beschäftigten gute Arbeitsbedingungen bieten, werden zukünftig gute Fachkräfte finden, so der Paritätische Wohlfahrtsverband in Nordrhein-Westfalen. In seinem Praxis-

\section{E-Mail aus Kampala}

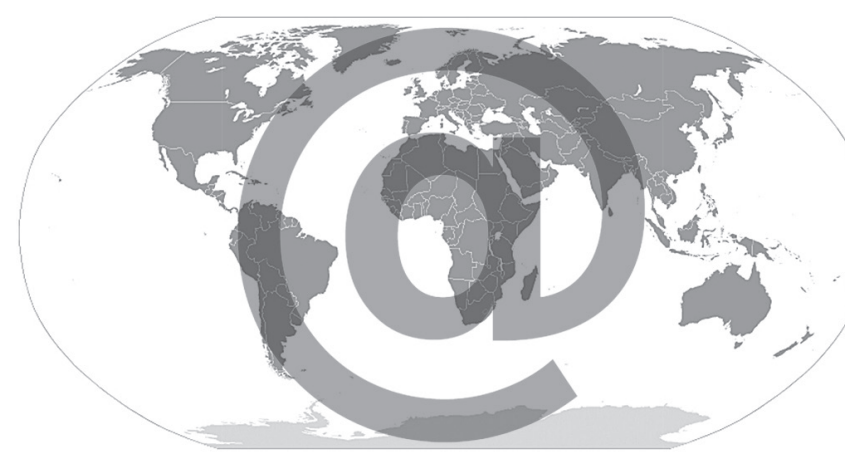

\section{Schanzenfest in Hamburg}

Am 28. September letzten Jahres sind Flüchtlinge, hauptsächlich aus Afrika und Asien, in Hamburg zusammen gekommen, um ihre Erfahrungen auszutauschen und mit interessierten Bürgerinnen und Bürgern zu reden. Auf dem Programm standen auch Musik, Kochen und verschiedene Workshops. Es war wie bei einem Festival und ich fühlte mich, als wäre ich wieder in Uganda. Ich sah viele Afrikaner, die kochten, miteinander sprachen und traditionelle afrikanische Musik machten. Das Ganze war ein wenig unwirklich für mich. Vor allem befremdete mich der Markt von gebrauchten Dingen, von Kleidung, Taschen, Schuhen, Büchern bis zu Elektrogeräten. Für die Käufer war offenbar das Gebot der Stunde, durch Verhandlungen einen möglichst niedrigen Preis zu erzielen. Da zog ich lieber weiter zu den anderen Aktivitäten auf diesem Teil des Hamburger Schanzenfestes. So traf ich einen nigerianischen Aktivisten der "Lampedusa-Gruppe". Er war Mitglied einer Protestgruppe von 300 Flüchtlingen, die im März 2013 nach Hamburg gekommen waren. Die meisten stammten aus Libyen und waren vor dem Bürgerkrieg aus ihrem Land geflohen. Sie hatten sich mit Booten über das Mittelmeer nach Lampedusa retten können. Ich hatte ein langes Gespräch, vor allem weil ich wissen wollte, wie Flüchtlinge in Deutschland leben. Berührt hat mich besonders die Mitteilung, dass einige von innen auf der Straße leben müssen und dass viele Flüchtlinge in Deutschland nicht arbeiten dürfen. Diese Menschen können deshalb kein eigenes Geld verdienen und kein normales Leben leben. Im Winter müssen sie oft andere fragen, ob sie bei ihnen übernachten können. Einige von ihnen können in Flüchtlingslagern leben, wo es aber kaum eine Privatsphäre gibt und die Sprachbarrieren besonders hoch sind, da die Bewohner meistens aus ganz verschiedenen Ländern und Kulturen kommen. Meine Gespräche vermittelten mir den Eindruck, dass Europa wirklich nicht das Paradies auf Erden ist, wie viele meiner Landsleute denken!

Onyango Aldo

Onyango Aldo war als Jugendlicher im Rainbow House of Hope Uganda, das von einer Nichtregierungs-Organisation getragen wird, die sich für die Verbesserung der sozialen Situation benachteiligter Kinder und Jugendlicher einsetzt. Er war im letzten Jahr aufgrund einer privaten Einladung in Deutschland.

www.rainbowhouse.info 


\section{Themenhefte der Blätter der Wohlfahrtspflege}

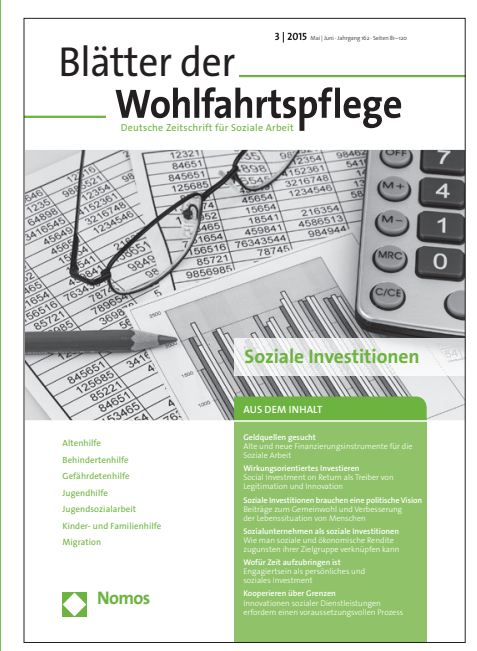

Schnittstellen der Jugendhilfe

Blätter der Wohlfahrtspflege 5/2007

Selbstständigkeit in der Sozialen Arbeit Blätter der Wohlfahrtspflege 6/2007

\section{Kindertagesstätten}

Blätter der Wohlfahrtspflege $1 / 2008$

Jugendsozialarbeit

Blätter der Wohlfahrtspflege 2/2008

Wohlfahrtsverbände

Produktionsauftrag:

zivilgesellschaftlicher Mehrwert

Blätter der Wohlfahrtspflege $3 / 2008$

Islam

Kritische Kooperation erwünscht:

Muslimische Vereine als Partner in der

Sozialen Arbeit

Blätter der Wohlfahrtspflege 4/2008

\section{Pflegeversicherung}

Fünf Fragen an die Pflege der Zukunft: Who cares? Wie Unterstützung und Kooperation gestalten? Wie Teilhabe sichern? Wie finanzieren? Was macht gute Pflege aus?

Blätter der Wohlfahrtspflege 5/2008

\section{Corporate Citizenship}

Gute Geschäfte: Die Renaissance unternehmerischen Engagements

Kooperationen sind Tauschgeschäfte: Wie man Unternehmen als Partner gewinnt Pflegeversicherung

Blätter der Wohlfahrtspflege $6 / 2008$

\section{Aspekte kommunaler Versorgung}

Die Zukunft der Kommune: weniger,

älter, bunter

Blätter der Wohlfahrtspflege 1/2009

\section{Forschung}

Wissen generieren für die Theorie und

für die Praxis Sozialer Arbeit

Blätter der Wohlfahrtspflege 2/2009

\section{Verbraucherschutz}

Vom Klienten zum Kunden - Konsequenzen

für soziale Dienste und Einrichtungen

Blätter der Wohlfahrtspflege 3/2009

Recht

Fachkräfte Sozialer Arbeit als Experten für soziales Recht

Blätter der Wohlfahrtspflege 4/2009

\section{Rechtsextremismus}

Freie Wohlfahrtspflege gegen pädagogische und soziale Bestrebungen rechtsextremer Organisationen

Blätter der Wohlfahrtspflege 5/2009

Gesundheitsschutz

Befähigung zur Gestaltung gesunder Lebenswelten

Blätter der Wohlfahrtspflege 6/2009

Ethik

Soziale Gerechtigkeit als Grundlage der

Profession Sozialer Arbeit

Blätter der Wohlfahrtspflege 1/2010

\section{Armutsberichterstattung}

Was mit dem ersten Armutsbericht von 1989 angestoßen wurde - und was immer noch nicht erreicht ist

Blätter der Wohlfahrtspflege 2/2010

\section{Beschäftigungsförderung}

Das Arbeitsleben mit Lebensarbeit verbinden und Verwirklichungschancen mehren Blätter der Wohlfahrtspflege 3/2010

Zentren und Netze der Kompetenz

"Nicht gegeneinander, sondern miteinander arbeiten"

Blätter der Wohlfahrtspflege 4/2010

Familienförderung und Kinderschutz Die Förderung menschlicher Beziehungen als Aufgabe von Wohlfahrt

Blätter der Wohlfahrtspflege 5/2010

Corporate Governance

Soziale Dienste und Einrichtungen gut und richtig führen

Blätter der Wohlfahrtspflege 6/2010

Care

Sorge mit Aussicht - der private Haus-

halt und die Soziale Arbeit

Blätter der Wohlfahrtspflege 1/2011

\section{Soziale Daseinsvorsorge}

Kommunen zwischen Haushaltskon-

solidierung und ihren Aufgaben als

Koordinator lokaler Arrangements

Blätter der Wohlfahrtspflege 2/2011

Generalisierung vs. Spezialisierung

Die soziale Profession braucht Einheit in

Vielfalt

Blätter der Wohlfahrtspflege 3/2011

\section{Quartiersmanagement}

Partizipation als Gewinn für alle Blätter der Wohlfahrtspflege 3/2011

\section{Innovationen}

Zwischen Reformstau und Reformmüdigkeit Blätter der Wohlfahrtspflege 5/2011

\section{Neuer Wohlfahrtsmix}

So viel Nachbarschaft wie möglich, so viel professionelle Hilfe wie nötig Blätter der Wohlfahrtspflege 6/2011

Person - Milieu - Sozialraum

Das Quartier als Milieu der Dienste und des Engagements

Blätter der Wohlfahrtspflege 1/2012

Versorgung gestalten

Vom Zusammenwirken von Professio-

nen, Diensten und Nutzern

Blätter der Wohlfahrtspflege 2/2012

Beratung in der Sozialen Arbeit

Querschnittsaufgabe und spezialisiertes

Arbeitsfeld

Blätter der Wohlfahrtspflege 3/2012

Lebensqualität

Eine über Wachstum und Wohlstand

hinausgehende gesellschaftliche Leitidee

Blätter der Wohlfahrtspflege 4/2012

Steuerung in der Sozialen Arbeit

Blätter der Wohlfahrtspflege 4/2012

Soziale Freiwilligendienste

Blätter der Wohlfahrtspflege 6/2012

Pflege

Blätter der Wohlfahrtspflege 1/2013

Wohnen in Bewegung

Blätter der Wohlfahrtspflege 2/2013

Personal in der Sozialen Arbeit

Blätter der Wohlfahrtspflege 3/2013

Orte früher Bildung

Blätter der Wohlfahrtspflege 4/2013

Europäische Union

Blätter der Wohlfahrtspflege 5/2013

Schule und Soziale Arbeit

Blätter der Wohlfahrtspflege 6/2013

Finanzierung Sozialer Arbeit

Blätter der Wohlfahrtspflege 1/2014

Dienste am Arbeitsmarkt

Blätter der Wohlfahrtspflege 2/2014

Soziale Medien und Soziale Arbeit

Blätter der Wohlfahrtspflege 3/2014

Freiwilliges Engagement

Blätter der Wohlfahrtspflege 4/2014

Migration heute

Blätter der Wohlfahrtspflege 5/2014

Freie Wohlfahrtspflege

Blätter der Wohlfahrtspflege 6/2014

Sterben, Tod, Trauer

Blätter der Wohlfahrtspflege 1/2015

40 Jahre Psychiatrie-Enquete

Blätter der Wohlfahrtspflege 2/2015

Soziale Investitionen

Blätter der Wohlfahrtspflege 3/2015

Jahresabonnement incl. Online 79,- $€$

(Privatbezieher); Studenten und arbeitslose Bezieher (jährliche Vorlage einer Bescheinigung erforderlich) $41,-€$; Institutionen incl. Online 119,- $€$; Einzelheft $18,-€$. Alle Preise verstehen sich incl. MwSt., zzgl. Vertriebskostenanteil. Nomos Verlagsgesellschaft, Postfach 1003 10, 76484 Baden-Baden, Telefon 07221 2104-O, Fax 07221 210427, E-Mail Nomos@nomos.de, Internet http://www.nomos.de 
handbuch »Gute Arbeit leben« bündelt er Tipps gegen den Fachkräftemangel speziell für Arbeitgeber aus der Sozialwirtschaft. Das Ziel: Soziale Betriebe wie die Kita, den Pflegedienst oder das Behindertenwohnheim als attraktiven Arbeitgeber etablieren. Das Praxishandbuch kann kostenfrei heruntergeladen werden.

www.gute-arbeit-leben.de

\section{Demokratie in den Städten}

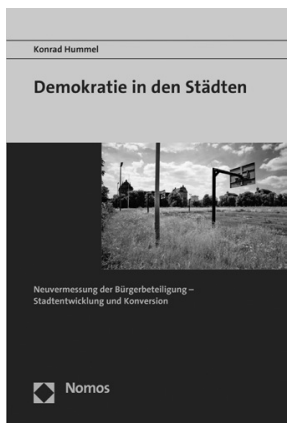

Das Buch von Konrad Hummel befasst sich sowohl konzeptionell mit den Fragen einer Bürgerbeteiligung in der Demokratie als auch mit der Einordnung eines mehrjährigen Stadtentwicklungsprozesses von ehemaligen Militärflächen in Mannheim. An vielen Aspekten wird aufgezeigt, wie sehr eine oft aufgeregte Bürgerbeteiligungsdiskussion vergisst, dass längst nicht alle Menschen unserer bunt und heterogen gewordenen Städten mitgenommen und aktiviert werden. Der Autor greift auf zahlreiche Erfahrungen sozialer Beteiligungsprojekte zurück, die er in den letzten zwei Jahrzehnten angeregt und begleitet hat und wirbt für eine differenzierte Teilhabestrategie statt oberflächlicher Abstimmungsmechanismen kleiner Gruppen.

Konrad Hummel:

Demokratie in den Städten.

Neuvermessung der

Bürgerbeteiligung - Stadtentwicklung und Konversion. Nomos

Verlagsgesellschaft, Baden-Baden

2015. 192 Seiten. 24,-Euro.

ISBN 978-3-8487-1785-9.

\section{Neues Internetportal "Gute Praxis» online}

Ein neues Online-Portal des REHADATInformationssystems will gelungene Beispiele sammeln, wie Menschen mit Behinderung erfolgreich arbeiten können. Es stellt zielgerichtete Informationen,

\section{Termine}

Übergänge in der Sozialen Arbeit. Internationaler Kongress der Schweizerischen Gesellschaft für Soziale Arbeit. 3. und 4. September 2015 in Zürich. www.project.zhaw.ch

Systemisches Denken und Handeln im Allgemeinen Sozialen Dienst. 7. und 8. September 2015 in Münster. www. weiterbildung-sozialwesen.de

Wirtschaftliche Steuerung sozialer Betriebe: Transparenz schaffen, Ressourcen managen. 7. bis 9. September 2015 in Remagen-Rolandseck. www. awo-bundesakademie.org

Sponsoring und mehr-Unternehmenskooperationen. 15. September 2015 in München. www.ibpro.de

Aktuelle Entwicklungen in der europäischen Sozialpolitik. 17. und 18. September 2015 in Erkner (bei Berlin). www. deutscher-verein.de

Unbegleitete volljährige Flüchtlinge den Übergang in die Selbstständigkeit erfolgreich gestalten. 17 . und 18 . September 2015 in Germerode (Nordhessen). www.igfh.de

Europa vor Ort: EU-Fördermittel für sozialwirtschaftliche Projekte. 17. und 18. September 2015 in Berlin. www. bfs-service.de

Führungsaufgabe "Gesundheit stärken«. 20. bis 25. September 2015 im Kloster Schöntal (Baden-Württemberg). www.paritaetische-akademie-nrw.de

Die gutachtliche Stellungnahme in der Sozialen Arbeit. 22. und 23. September 2015 in Münster. www.weiterbildungsozialwesen.de

Lobbying und Interessenvertretung: Benachteiligten eine Stimme geben. 21. bis 23. Oktober 2015 in Frankfurt am Main. www.bundesakademie-kd.de

Professionelle Fördermittelakquise für Organisationen der Sozialwirtschaft. 30. September 2015 in Hamburg. www.bfs-service.de

Politik der Verhältnisse, Politik des Verhaltens: Widersprüche der Gestaltung Sozialer Arbeit. Bundeskongress Soziale Arbeit 2015. 30. September bis 2 . Oktober 2015 in Darmstadt

Freiwilligenmanagement in der Sozialen Arbeit. 5. Oktober 2015 in Münster. www.weiterbildung-sozialwesen.de
Von der Fach - zur Führungskraft. 5. bis 8. Oktober 2015 in Remagen-Rolandseck. www.awo-bundesakademie.org

Forum Quartiermanagement. Interdisziplinäre Fortbildung für Quartierakteure zur Aktivierung von Nachbarschaften und Projekte. Fortbildung in drei Modulen. Beginn 7. bis 9. Oktober 2015 in Frankfurt am Main. www.ba-kd.de

Rehacare 2015. Fachmesse mit Kongress. 14. bis 17. Oktober 2015 in Düsseldorf. www.rehacare.de

... und plötzlich bin ich Leitung: Von der Fach- zur Führungskraft. Fit für den Start als Vorgesetzte und Vorgesetzter. Zwei Kursteile: 20. bis 23. Oktober 2015 und 16. bis 19. Februar 2016, jeweils in Berlin.

ConSozial 2015: Fachmesse mit Kongress. 21. und 22. Oktober 2015 in Nürnberg. www.consozial.de

Soziale Arbeit im Gesundheitswesen: Menschen erreichen, Teilhabe ermöglichen. Bundeskongress der Deutschen Vereinigung für Soziale Arbeit im Gesundheitswesen e. V. 5. und 6. November 2015 in Münster. www.dvsg.org

Facebook, Twitter \& Co. Der Einsatz von Social Media in gemeinnützigen Einrichtungen. 6. November 2015 in Berlin. www.akademie.org

Spendenrecht und Rechnungslegung für Fundraiser und Spendensammler. 9. November 2015 in Köln. www.bfs-service.de

Die Jugendhilfe in der Praxis. Aktuelle Fragen, Ausblick, Rechtsentwicklungen. 12. und 13. November 2015 in Berlin. www.kbw.de

Die Jugendhilfe in der Praxis. Ausblick Rechtsentwicklungen - Praxisfragen. Fachtagung Jugendhilfe Berlin. 12. und 13. November 2015 in Berlin. www. jugendhilfetagung.de

Das 5-Minuten-Gespräch. Kommunikation im Arbeitsalltag. 17. und 18. November 2015 in Stuttgart. www. wohlfahrtswerk.de

Rechtsfragen zur Vereinsführung: Schwerpunkt Haftung. 18. November 2015 in Köln. www.paritaetische-akademie-nrw.de

Aktuelle fachliche, fachpolitische und rechtliche Entwicklungen in der Sozialhilfe. 25. bis 27. November 2015 in Berlin. www.deutscher-verein.de 
Maßnahmen, Integrationsvereinbarungen und Aktionspläne zu einer inklusiven Arbeitswelt vor. Einen Überblick über die Inhalte von derzeit 900 Praxisbeispielen kann sich der Nutzer über die thematische Navigation verschaffen: entweder über die Art der Behinderung (deren Auswirkungen im Arbeitsleben kurz beschrieben sind) oder die durchgeführte Maßnahme (z. B. Ausbildung, Arbeitsgestaltung, Mobilität, Existenzgründung). Ergänzend steht eine Detailsuche zur Verfügung, mit der man Beispiele auch mithilfe von Schlagworten, Beschäftigungsbereichen oder Einschränkungen suchen kann. Das Portal bietet außerdem 145 nach Branchen sortierte, anonymisierte Integrationsvereinbarungen an, die zum Abschluss einer eigenen Vereinbarung anregen sollen. Dabei handelt es sich um Einzelvereinbarungen für Betriebe und Dienststellen oder um Gesamtvereinbarungen für Konzerne oder Obere Behörden. Die Informationen werden laufend aktualisiert und ergänzt. REHADAT ist ein Projekt des Instituts der deutschen Wirtschaft Köln und wird gefördert vom Bundesministerium für Arbeit und Soziales.

www.rehadat.de

\section{Neues Konzept für}

\section{Beschwerdemanagement} im Betreuungswesen

Der Bundesverband der Berufsbetreuerinnen und Berufsbetreuer reformiert sein Beschwerdemanagement. Regionale Schlichterinnen und Schlichter sollen künftig als vorgelagerte Instanz für die zentrale Beschwerdestelle in Hamburg aktiv Lösungen mit den Konfliktparteien erarbeiten. Ziel ist es, die Qualität von Betreuung zu verbessern, indem Beschwerden zügig und in der Region bearbeitet werden. Jede Klientin und jeder Klient kann sich an die Beschwerdestelle wenden, wenn er oder sie unzufrieden mit seinem oder ihrem Betreuer ist. Meist geht es um finanzielle Angelegenheiten. Gebraucht werden eine gleich zu Beginn des Beschwerdeverfahrens einsetzende fachlich orientierte Schlichtung zwischen den Parteien. Der Bundesverband der Berufsbetreuerinnen und Berufsbetreuer e. V. (BdB) zählt mehr als 6.500 Mitglieder.

http://bdb-ev.de

\section{Kennzahlen}

\section{"Das Teuflische an den Zablen ist, dass sie so einfach sind."}

Hans Magnus Enzensberger, deutscher Schriftsteller (geb. 1929)

\subsection{0 .000}

Zahl der Erwerbstätigen in Deutschland, die zwei oder mehr Tätigkeiten nachgehen

(Arbeitskräfteerhebung 2014)

\subsection{4 .620}

Zahl der Sanktionen durch deutsche Jobcenter im Jahre 2014

\subsection{0}

Zahl der Kirchengebäude und Kapellen im Besitz der Evangelischen Kirche in Deutschland

(Erhebung 2010)

\subsection{7}

Zahl der Kinder und Jugendlichen zwischen 10 und 19 Jahren, die 2013 wegen akuten Alkoholmissbrauchs stationär in einem Krankenhaus behandelt werden mussten

\subsection{6}

Zahl der Bezieher von Hilfe zum Lebensunterhalt (SGB XII) in Deutschland außerhalb von Einrichtungen

(Stand 31.12.2013)

\subsection{9}

Zahl der Bezieher von Hilfe zum Lebensunterhalt (SGB XII) in Deutschland in Einrichtungen

(Stand 31.12.2013)

\subsection{3}

Zahl der Inobhutnahmen (\$ 42 SGB VIII) deutscher Jugendämter im Jahre 2013 